\title{
A Study of Detection of Drowsiness and Awakeness using Non-contact Radar Sensors
}

\author{
In Chung Kyo ${ }^{1}$ and Min Byung Chan ${ }^{2}$ \\ ${ }^{1}$ Student, Industrial Management Engineering, Hanbat National University, Korea, inboss@ gmail.com \\ ${ }^{2}$ Professor, Industrial Management Engineering, Hanbat National University, Korea, bcmin@ hanbat.ac.kr \\ *Correspondence: Min Byung Chan; Email: bcmin@hanbat.ac.kr
}

\begin{abstract}
Biometric information is used in a variety of industrial fields. Heart rate and respiration rate, in particular, are widely applied not only in medical institutions but also in life safety. However, a sensor must be worn or directly attached to the human body to obtain a bio signal, which is inconvenient and limits its application. In this study, a $24 \mathrm{GHz}$ radar sensor module is developed, and an algorithm is implemented by analyzing the frequency and peak values of a human participant's heartbeat and respiration signals in an unconstrained state. In the experiment, the existing ECG equipment (MP150) and radar sensor module are compared. The results indicate that the average value of MP150 is higher than that of the Doppler sensor in terms of all parameters; however, the deviation of the Doppler sensor is small, and the bias is low. Furthermore, it is confirmed that the HRV decreases in the drowsy state compared to that in the wakeful state in both devices. These results confirm that bio-signals change during drowsiness, and conversely, drowsiness can be detected through changes in bio-signals, which is a significant finding.
\end{abstract}

Keywords: Drowsiness, Non-contact, Vital-signal, Radar sensor, ECG.

\begin{tabular}{|c|c|}
\hline \multicolumn{2}{|l|}{ ARTICLE INFORMATION } \\
\hline \multicolumn{2}{|c|}{$\begin{array}{l}\text { Author(s): In Chung Kyo and Min Byung Chan } \\
\text { Received: } 25 \text { Sep, 2021; Accepted: } 20 \text { Dec, 2021; Published: } 13 \text { Jan, 2022; }\end{array}$} \\
\hline $\begin{array}{l}\text { e-ISSN: } 2347-470 X ; \\
\text { Paper Id: IJEER-110319; }\end{array}$ & Crossef member \\
\hline $\begin{array}{l}\text { Citation: doi.org/10.37391/IJEER.090303 } \\
\text { Webpage-link: }\end{array}$ & 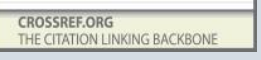 \\
\hline
\end{tabular}

\section{INTRODUCTION}

In the past five years, accidents caused by driver drowsiness have been increasing, accounting for more than $20 \%$ of all accidents. Drowsiness is induced when a person is physically or mentally tired and in is a state close to sleep. It is an involuntary response signaling the body to rest. The primary factor that induces drowsiness is the secretion of a sleep hormone called melatonin. Drowsiness is also induced when a person is tired or hungry, the concentration of carbon dioxide in the air is high, or the weather is warm.

Drowsiness can also occur when performing repetitive and focused tasks, such as driving, particularly on highways where driving conditions are rather monotonous [2]. The nature of highways makes driving at high speeds significantly risky, and accidents resulting from drowsy driving can cause severe personal injury. According to Korean Road Traffic Authority data, approximately $23 \%$ of highway accidents over the past 10 years have been caused by drowsy driving, with the percentage steadily increasing.

Therefore, many studies are being conducted to prevent drowsy driving and industrial accidents by detecting drowsiness in advance while driving or working.
Drowsy driving can be detected through three methods. In the first method, drowsiness can be detected based on the degree of lane departure and degree of steering wheel manipulation. The second method is based on the behavioral state of the driver, wherein drowsiness can be detected by analyzing the facial behavior of the driver such as eyelid closure, pupil size, and head movement, through camera image processing. In the third method, drowsiness is detected based on bio-signals, and research is underway to determine whether drowsiness can be detected through heart rate or respiration.

The detection of drowsiness based on bio-signals utilizes an electrocardiogram and an EEG, and many studies have been conducted on this method of detection relatively recently. Although this detection method is accurate, because electrodes need to be directly attached to the body of the driver, it causes inconvenience for the driver and has disadvantages in conscious judgment.

Therefore, in this study, the correlation between the accuracy of the existing contact method and the electrocardiogram is investigated through the detection of drowsiness based on biosignals in the non-contact state. This study aims to measure the electrocardiogram using a non-contact Doppler radar sensor. The Doppler radar can be used to measure a reflected signal by matching a microwave signal to a target, and receiving a signal whose phase is modulated according to the movement of the target.

Many studies have been conducted to detect heart rate and respiration using the Doppler radar method. However, research on detecting drowsiness using the Doppler radar method is insufficient. Therefore, we intend to design and measure a radar system to prevent the driver from feeling drowsy while driving using the Doppler radar method. 


\section{[Fig. 2] Doppler Radar Module Used for Testing}

\section{METHOD}

To detect drowsiness presented in the introduction, we will manufacture a Doppler-based radar sensor module that will be tested, and conduct tests on subjects to analyze the heart rate, HRV, and respiratory rate of awakening and drowsiness to check whether drowsiness is detected.

\subsection{Research Objectives}

A radar (radio detection and ranging) is a wireless device that emits radio waves to detect targets, the distance from a target, and a state, among others, based on the reflected waves. Radars enable the detection of various types of information that cannot be confirmed by humans at a distance due to geographical distances, and suitable responses can be determined based on the results. To implement a radar function that detects various information, it is necessary to use a wideband frequency that can contain a large amount of information, and transmit and receive signals of repetitive pulses. With the development of digital signal processing and RF technology, radar sensing technology using an impulse with ultra-wide frequency characteristics has led to a rapid technological advancement. Therefore, the applications of radar technology are also expanding to various fields such as healthcare, national defense, and life safety. This study aims to detect drowsiness by extracting respiration and heart rate data by combining the Doppler radar technology with a non-contact biosignal sensor.

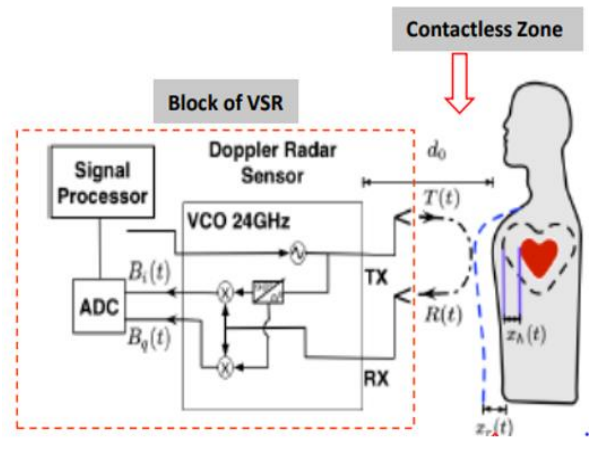

[Fig. 1] Radar Radio Wave Measurement Configuration

\subsection{Doppler radar specifications used in the test}

A non-contact radar-based sensor was used for non-contact vital sign monitoring. The radar sensor could detect and measure movements of less than $1 \mathrm{~mm}$ on the skin surface of the chest wall caused by the heartbeat.

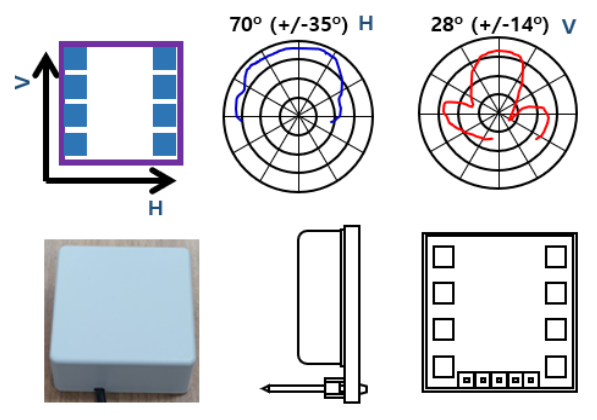

The Doppler radar sensor module used in this test was a $24 \mathrm{GHz}$ band Doppler radar sensor module that could remotely measure bio-signals. An RF front-end module was developed using a commercial chipset, planar passive element, and microwave antenna in consideration of the radio wave attenuation characteristics in the body according to the operating frequency characteristics. Algorithm was applied.

The bio-signals acquired through the radar were calculated and displayed as respiration and heart rate data through signal processing.

This sensor module collected data by detecting minute movements of the human body, such as heartbeat, and the accuracy varied depending on the distance; thus, the experiment was conducted within 1.5 to $3 \mathrm{~m}$.
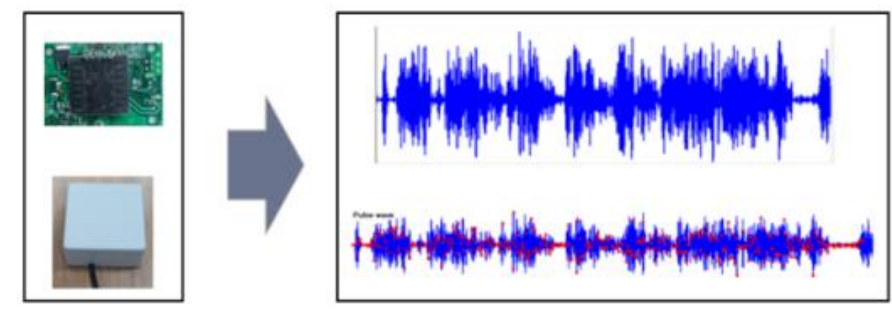

[Fig. 3] Doppler Radar Sensor Module and Signal Types

\subsection{Signal processing process}

The development of the RF module and interface part is output and input signal processing for analog baseband signal of RF.

$\mathrm{ADC} / \mathrm{DAC}$ and a digital interface between ADC/DAC and MCU to transfer analog baseband signals to the digital domain were implemented.

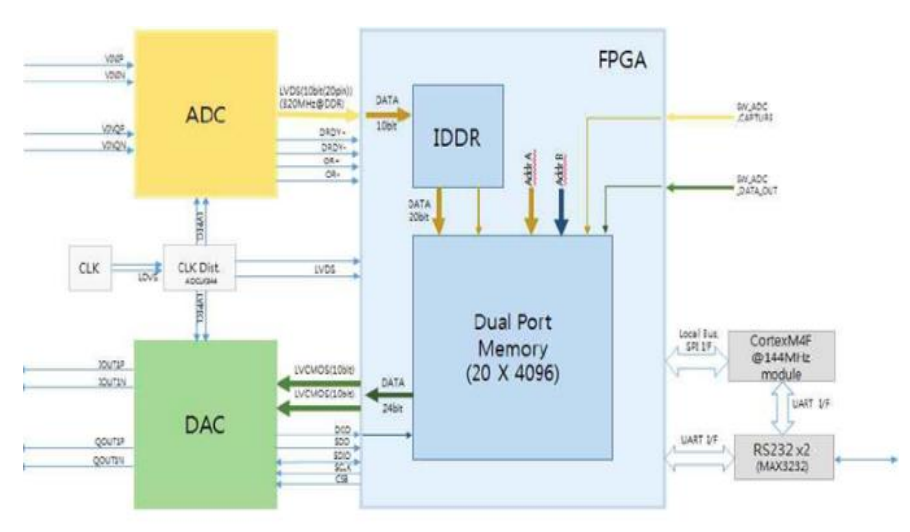

[Fig. 4] ADC/DAC Interface Configuration Using FPGA

Because the accuracy of the radar sensor is vital in this test, an appropriate frequency setting and the ability to remove noise are required.

Therefore, the frequency was set to $24 \mathrm{GHz}$ in the most optimized section, and a filter for ambient noise was also developed to minimize noise. 
The signal collected from the radar sensor for a certain duration was typically displayed in the form of a frequency through fast-Fourier transform (FFT), and the heart rate was $50-90$ beats/min and respiration rate was $12-48$ beats $/ \mathrm{min}$.

By converting this into a frequency band, the heart rate appeared in the frequency band of $0.8-1.5 \mathrm{~Hz}$ and respiration occurred in the frequency band of $0.2-0.8 \mathrm{~Hz}$. In the conventional method, an accurate peak can be obtained as the number of data having periodicity increases owing to the FFT characteristics.

However, because the human heart rate includes human specificity and does not have complete periodicity depending on characteristics and conditions, there is a limit to representing it as a single peak.

Therefore, acquisition of bio-signal variation information that varies with time can serve as an indicator based on sympathetic-parasympathetic activation and contains more human body information than the heart rate per minute. Hence, it is necessary to process the sensor output in the time domain to obtain bio-signal characteristics.

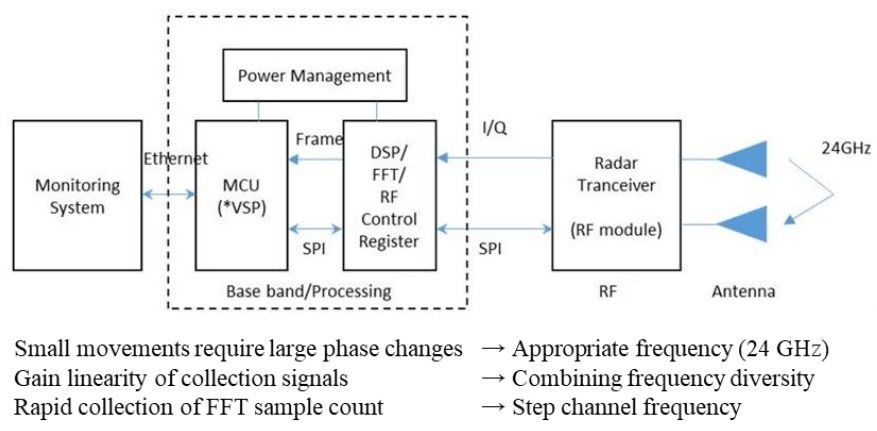

[Fig. 5] Rader Bio-signal Processing System Diagram

\subsection{Threshold-voltage-based radar sensor data extraction algorithm}

To detect a bio-signal (heart rate per minute) with a low signal-to-noise ratio and high accuracy, an algorithm was developed using a threshold-voltage-based detection technique. The characteristics of the original signal collected from the radar sensor were continuously varied by disturbance and clutter, requiring the threshold voltage for detecting the heartbeat peak to be changed accordingly to obtain a high detection accuracy. Based on previously collected data, a heartbeat peak detection algorithm that adaptively changes the threshold voltage was studied, and an algorithm that can improve the bio-signal detection accuracy using this was implemented as follows.

\subsection{Radar transceiver}

To secure the system resolution, the phase noise specification of VCO/PLL must be secured to the maximum extent possible.

In particular, the mismatch between the DC offset and IQ signal in the digital domain was corrected by applying an optimization structure, such as an image rejection structure, to exclude the image signals.

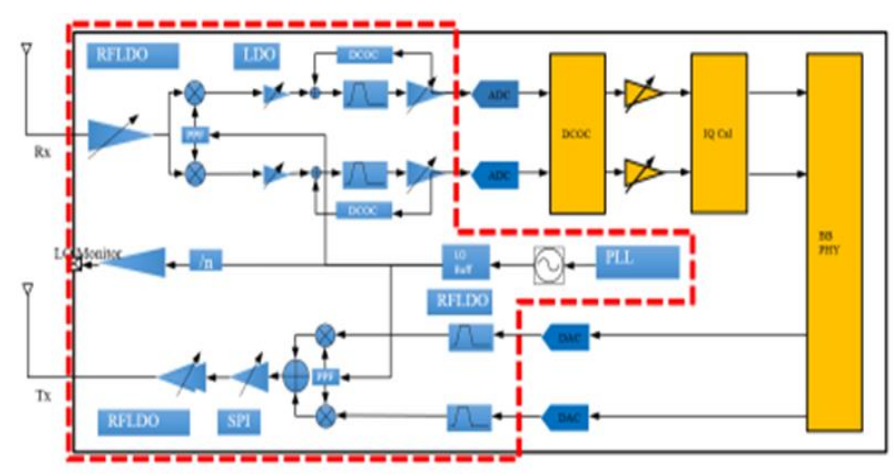

[Fig. 6] Block Diagram of Non-Contact Bio-signal Radar Module

\subsection{Power module for supplying power to RF module}

The buck converter and boost converter cannot provide the required voltage independently; even if these two converters are used together, an operation gap may occur in the output voltage range due to the limitation of the control pulse duty ratio operation of each converter. Thus, a tri-mode buck-boost DC-DC conversion module with a conversion function was constructed.

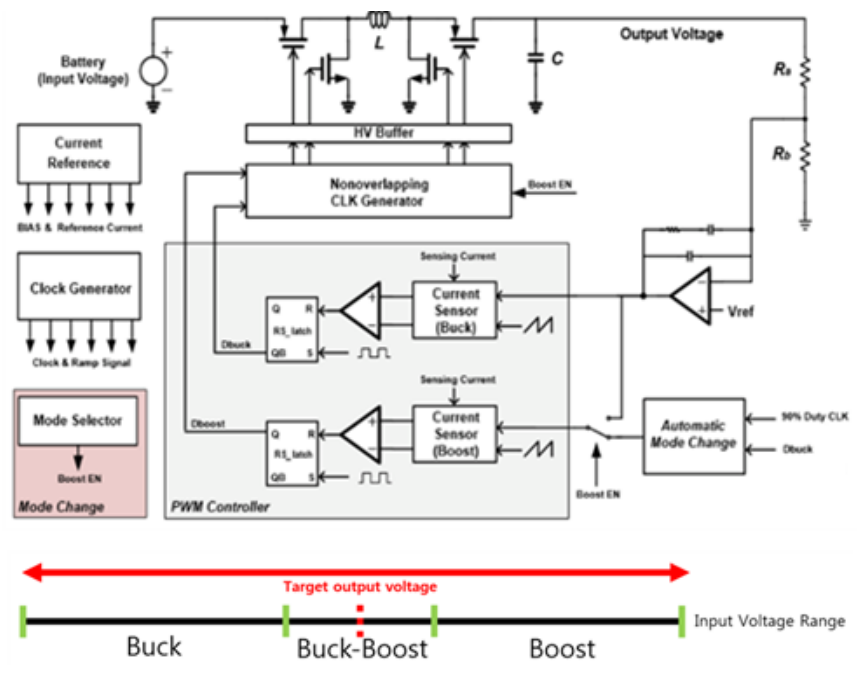

[Fig. 7] Mode Change of Tri-Mode Buck-Boost Converter

Low-noise characteristics are crucial for the power supply of signal amplifiers and high-resolution ADCs; therefore, a lownoise regulator module as illustrated in Figure 7 was implemented. To ensure low-noise characteristics, power switch control with standard deviation (SD) modulation was implemented, and a boost function to dynamically increase the loop GBW was implemented to maintain the regulation performance at a low-voltage dropout.

\subsection{Signal path and RF board}

The board design and RF module were manufactured considering the reflection coefficient of RF input and output. In addition, the design considered the arrangement of the RF and analog signal paths so that the RF signal is not affected by 
the digital signal of the platform board. Moreover, when mounting on the platform board, the RF element was placed in consideration of the actual antenna arrangement and signal direction.
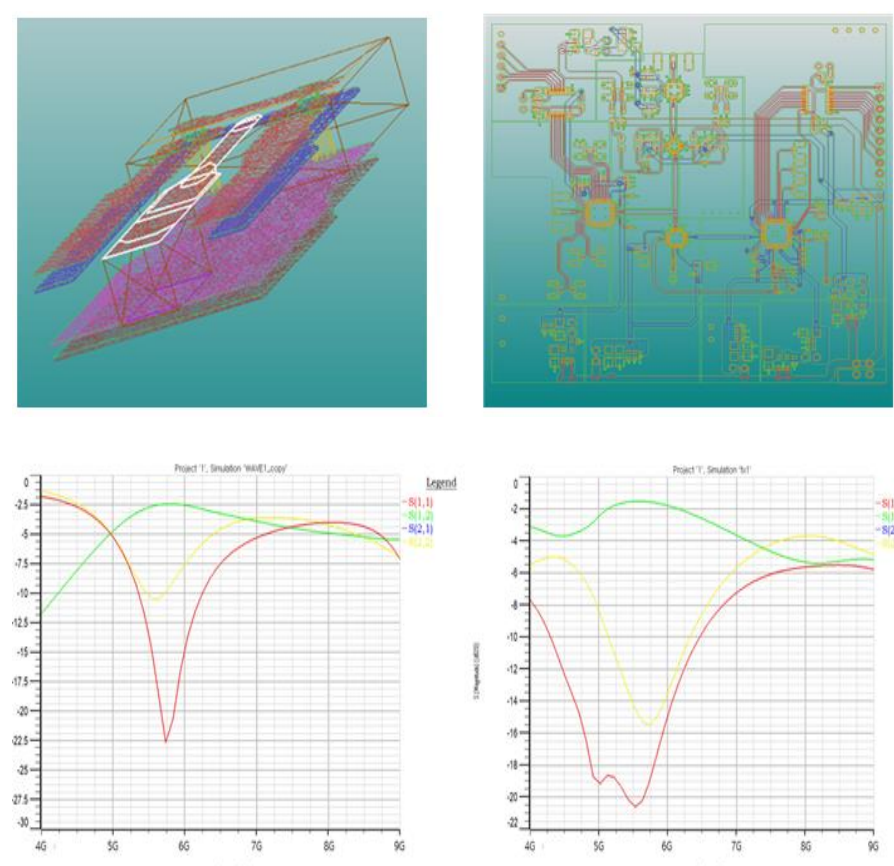

[Fig. 8] Simulation of PCB layout matching characteristics (Sparameter)

\subsection{Subjects}

Ten healthy men and women in their twenties, who had never had any heart disease, voluntarily participated in the experiment. The average height, weight, and BMI of the subjects were $168.97 \mathrm{~cm}, 80.82 \mathrm{~kg}$, and $28.66 \mathrm{~kg} / \mathrm{m} 2$, respectively (Table 2). Subjects were instructed to refrain from consuming caffeine, drinking alcohol, smoking, etc., which could affect the ECG measurements, on the day before and on the day of the experiment, and to get more than $7 \mathrm{~h}$ of sleep.

[Table 1] Subject information

\begin{tabular}{|c|c|c|c|}
\hline $\mathrm{N}=10$ & Height $(\mathrm{cm})$ & Weight $(\mathrm{kg})$ & $\mathrm{BMI}\left(\mathrm{Kg} / \mathrm{m}^{2}\right)$ \\
\hline mean & 168.97 & 80.82 & 28.66 \\
\hline $\mathrm{SD}$ & 8.59 & 28.53 & 7.42 \\
\hline
\end{tabular}

\subsection{Test environment and procedure}

In this test, the temperature was $27.0 \pm 3.0^{\circ} \mathrm{C}$, the humidity was $57.0 \pm 2.0 \%$ R.H., and the illuminance was $21.0 \pm 3.0$ lx (Table 2).

[Table 2] Laboratory environment

\begin{tabular}{|c|c|c|}
\hline Temperature $\left({ }^{\circ} \mathrm{C}\right)$ & Humidity (\% R.H.) & Illumination (lx) \\
\hline $27.0 \pm 3.0$ & $57.0 \pm 2.0$ & $21.0 \pm 3.0$ \\
\hline
\end{tabular}

[Table 3] Equipment used in the test

\begin{tabular}{|c|c|c|c|}
\hline $\begin{array}{c}\text { Equipment } \\
\text { name }\end{array}$ & $\begin{array}{c}\text { Equipment } \\
\text { image }\end{array}$ & Signal example & Note \\
\hline BioPac \\
MP150 \\
(BIOPAC \\
Systems Inc.)
\end{tabular}

A Doppler radar sensor was placed on a desk and the ECG and respiration rate were measured for $10 \mathrm{~min}$. In addition, biosignals were measured with a BioPac device. After measuring the arousal state signal for $10 \mathrm{~min}$, an eye patch and earplugs were worn by the subjects for $20 \mathrm{~min}$, who were then asked to lie down on a bed to induce sleep. After $20 \mathrm{~min}$, the subjects got up and the ECG and respiration rate were immediately measured in the same manner as in the awake state. Further, bio-signals were measured using the BioPack device.

The electrode position during ECG measurement through Biopac is shown in Fig. 9. The negative electrode was attached at the right side of the chest, the positive electrode was attached to the left side of the abdomen, and the ground electrode was attached to the left side of the chest. The sampling rates of both the Doppler racer sensor and Biopac MP150 were set to $200 \mathrm{~Hz}$.
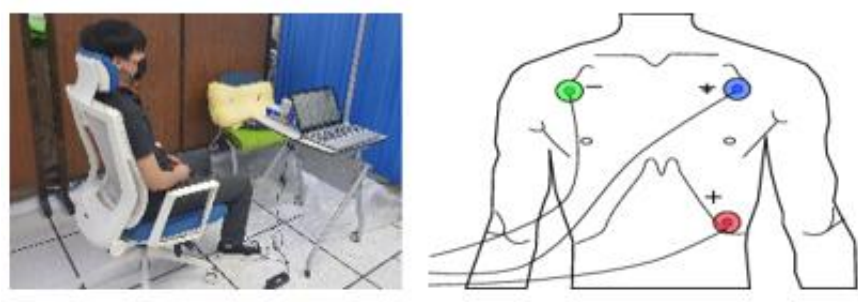

[Fig. 9] Position of ECG electrodes

\section{RESULTS}

Non-contact bio-signal measurements have been conducted through radar sensor module production. The transmission and reception distance of radar radio waves was able to bring the most accurate data between 1 to 3 meters, and the accuracy decreased as the distance increased. The difference in accuracy could also be confirmed according to the direction of the radar antenna. In other words, more accurate data could be obtained only when the radar antenna direction was directed to the subject's heart and tested. Since radar-type radio waves are affected by the surrounding environment, noise could be minimized by applying a threshold 
voltage in this study. In the results, the Doppler sensor and MP150 were compared, respectively, to analyze the measurement data results, and a significant difference in sleepiness was obtained in a non-contact method.

\subsection{Comparison of Doppler and MP150}

As shown in [Table 4], the average of the heart rate, HRV, and respiration measurement results of the MP150 are higher than those of the Doppler sensor. Furthermore, the SD of the Doppler sensor was smaller than that of MP150. The results of the t-test showed that the difference between the two devices has a significance level of $1 \%$, as shown in [Table 5].

[Table 4] Descriptive statistics for Doppler \& MP150

\begin{tabular}{|c|c|c|}
\hline$($ Mean \pm SD) & Doppler & MP 150 \\
\hline Heart rate & $68.39 \pm 7.46$ & $75.32 \pm 13.48$ \\
\hline HRV $(\%)$ & $61.25 \pm 7.87$ & $67.37 \pm 14.84$ \\
\hline Respiration & $16.49 \pm 4.68$ & $19.46 \pm 8.16$ \\
\hline
\end{tabular}

[Table 5] Doppler \& MP150 t-test results

\begin{tabular}{|c|c|c|c|c|}
\hline $\begin{array}{c}\text { (Doppler-MP } \\
\text { 150) }\end{array}$ & MD & df & $t$ & $\mathrm{p}$ \\
\hline Heart rate & -6.93 & 19 & -3.07 & $.001^{* *}$ \\
\hline HRV (\%) & -6.11 & 19 & -2.91 & $.004^{* *}$ \\
\hline Respiration & -2.98 & 19 & -1.61 & $.002^{* *}$ \\
\hline
\end{tabular}
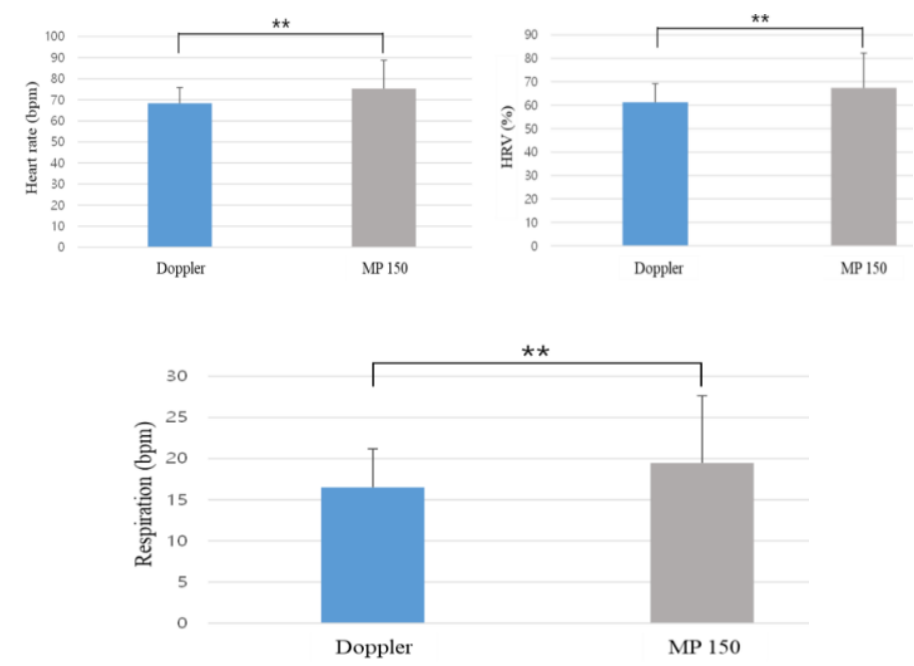

[Fig. 10] Comparison of equipment for heart rate and respiration

\subsection{Comparison of wakefulness and drowsiness}

The observation results of the HRV changes of the Doppler sensor and MP150 during the awake and drowsy states showed that both devices displayed a decreasing trend during drowsiness, as shown in [Table 6]. As shown in [Table 7], according to the t-test results, the HRV decreased during drowsiness than in the awake state, and a statistically significant difference was recognized with the significance level of $1 \%$.

[Table 6] Descriptive statistics for wakefulness and drowsiness

\begin{tabular}{|c|c|c|}
\hline$($ mean \pm SD $)$ & Doppler & MP 150 \\
\hline Awakening & $66.25 \pm 4.77$ & $72.76 \pm 6.74$ \\
\hline Drowsy & $56.26 \pm 4.31$ & $61.87 \pm 5.94$ \\
\hline
\end{tabular}

[Table 7] Wakefulness and drowsiness t-test results

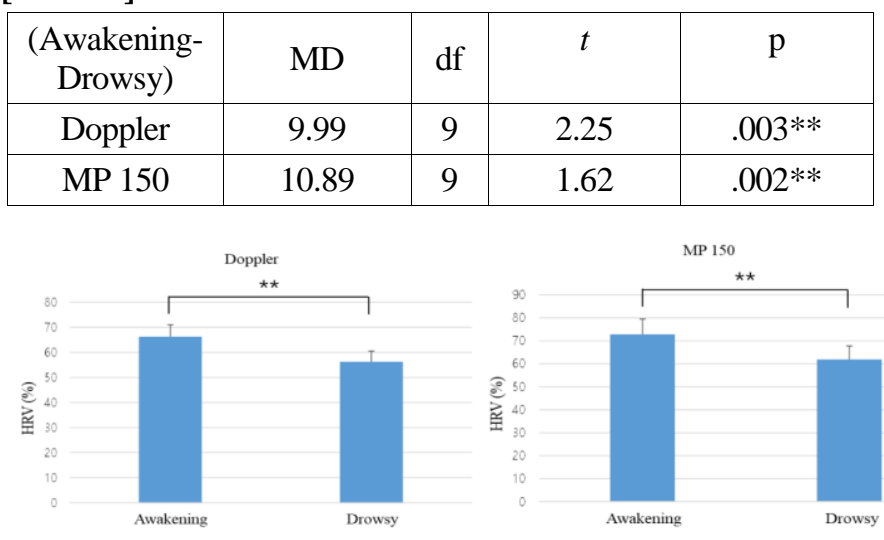

[Fig. 11] Comparison of equipment for heart rate

\section{DISCUSSION}

Various studies, such as face recognition, brain wave analysis, and radar methods, have been conducted to detect drowsy driving, with some regarding it as a technology with a transitional nature.

If autonomous driving technology is advanced in the future, a driver's condition will not affect safety.

Therefore, the concept of the driver itself disappears. Lanemaintaining assistance and emergency automatic braking systems, which are part of autonomous driving technology, have been introduced, to help prevent accidents.

However, the cost aspect of reducing redundant investment by introducing a higher level of technology cannot be ignored.

In this study, by applying radar technology, bio-signals could be analyzed in a noncontact manner and the awake state of the driver could be determined.

In particular, in this study, ECG parameters (heart rate, HRV, respiration) were measured and compared with the existing electrocardiogram (MP 150) and noncontact radar sensor (Doppler). Two major methods were considered for comparison: the comparison between devices and the comparison during wakefulness and drowsiness. In the case of comparison between devices, the average values of all the parameters for the MP150 were higher than those of the Doppler sensor; however, the variation of the Doppler sensor was small and its variability was low. In the case of comparison between wakefulness and drowsiness, HRV decreased in the drowsy state compared to the 
awake state in both devices. These results indicate that a change in the bio-signal occurs during drowsiness, and conversely, drowsiness can be detected through a change in the bio-signal. In the future, it is necessary to study whether the accuracy of the result data is improved by increasing the Doppler radar antenna as well as the frequency band and correlation with the measurement distance to analyze its use in the medical and remote monitoring.

In this study, to detect and prevent drowsiness, we proposed a radar sensor that measures respiration and heart rate in a noncontact manner using the Doppler radar method that combines the advantages of existing measurement methods. As a result of the experiment, values for heart rate, stress level, drowsiness, concentration, and mental fatigue could be checked in real time, and the average value of the above values could also be checked. However, it is thought that a clearer interpretation of what the level of drowsiness and concentration means is needed.

Applying to the medical field is expected to solve a number of problems in previous studies in that it does not directly contact the body. Existing contact-type research methods are limited to use in patients such as premature babies, burns or skin problems, or using multiple contact-type sensors is expected to solve these problems in making accurate diagnosis difficult in the case of sleep diagnosis. In addition, it is expected to lead to the development of medical devices that can be used on a daily basis outside of hospitals, given that they can continuously acquire biometric information while imposing less restrictions on subjects from a distance. This not only helps with a more accurate diagnosis, but can also be used for the purpose of immediately detecting and coping with risk signs such as sudden death prevention and neonatal sudden death syndrome of the elderly living alone.

\section{CONCLUSION}

With simple hardware configuration can measure a non-contact bio-signal. A possible $\mathrm{GHz}$ band Doppler radar sensor system is proposed. Depending on the operating frequency characteristics of the proposed radar sensor system. Considering the characteristics of attenuating radio waves in other bodies, a commercial chipset and a planar type.

RF Front-end modules were manufactured using passive elements, antennas, etc.

The non-contact radar sensor device proposed in this study confirmed the measurement accuracy of more than $90 \%$ compared to the conventional contact type device through comparative measurement of the 20 subjects.

Due to the characteristics of the original signal of the radar sensor, although it is difficult to secure additional information acquisitionenabled HRV analysis at the ECG level, HRV analysis accuracy is expected to increase in the future by applying signal processing techniques based on nonlinear algorithms.

\section{ACKNOWLEDGMENTS}

This research was supported by the research fund of Hanbat National University in 2021.

\section{REFERENCES}

[1] Kim, J.-Y.; Park, J.-H.; Jang, S.-Y.; Yang, J.-R. Peak Detection Algorithm for Vital Sign Detection Using Doppler Radar Sensors. Sensors 2019.

[2] Leem, S.K.; Khan, F.; Cho, S.H. Vital Sign Monitoring and Mobile Phone Usage Detection Using IR-UWB Radar for Intended Use in Car Crash Prevention. Sensors 2017, 17, 1240.

[3] Multimedia Tools \& Applications . Jul 2020, Vol. 79 Issue 25/26, p17793-17815. 23p

[4] Siddiqui, H.U.R.; Saleem, A.A.; Brown, R.; Bademci, B.; Lee, E.; Rustam, F.; Dudley, S. Non-Invasive Driver Drowsiness Detection System. Sensors 2021, 21, 4833.

[5] Smita S. Gajare, Dipti Y. Sakhare (2016), Analysis of Intra-body Communication Measurement in Biomedical Applications. IJEER 4(2), 53-56. DOI: 10.37391/IJEER.040203.

[6] Wang, D.; Yoo, S.; Cho, S.H. Experimental Comparison of IRUWB Radar and FMCW Radar for Vital Signs. Sensors.

[7] B. Alshaqaqi, A. S. Baquhaizel, M. E. Amine Ouis, M. Boumehed, A. Ouamri and M. Keche, "Driver drowsiness detection system," 2013 8th International Workshop on Systems, Signal Processing and their Applications (WoSSPA), 2013, pp. 151-155, doi: 10.1109/WoSSPA.2013.6602353

[8] Acharya UR, Joseph KP, Kannathal N, Lim CM, Suri JS (2006) Heart rate variability: a review. Med Biol Eng Comput 44(12):1031-1051

[9] Bailón R, Mainardi L, Orini M, Sörnmo L, Laguna P (2010) Analysis of heart rate variability during exercise stress testing using respiratory information. Biomed Signal Process Control 5(4):299-310

[10] Mateo J, Laguna P (2003) Analysis of heart rate variability in the presence of ectopic beats using the heart timing signal. IEEE Trans Biomed Eng 50(3):334-343

[11] Patel M, Lal S, Kavanagh D, Rossiter P (2011) Applying neural network analysis on heart rate variability data to assess driver fatigue. Expert Syst Appl 38(6):7235-7242

[12] Shinar Z, Akselrod S, Dagan Y, Baharav A (2006) Autonomic changes during wake-sleep transition: a heart rate variability based approach. Auton Neurosci 130:17-27

[13] Vicente J, Laguna P, Bartra A, Bailón R (2011) Detection of driver's drowsiness by means of hrv analysis. Comput Cardiol.

[14] Willis D, Waller P, Stutts J, Roth T (1998) Drowsy driving and automobile crashes. Tech Rep DOT HS 808 707, National Highway Traffic Safety Administration.

[15] Yang G, Lin Y, Bhattacharya P (2010) A driver fatigue recognition model based on information fusion and dynamic bayesian network. Inf Sci (Ny) 180(10):1942-1954

[16] Chin-Teng Lin, Ruei-Cheng Wu, Sheng-Fu Liang, Wen-Hung Chao, Yu-Jie Chen and Tzyy-Ping Jung, "EEG-based drowsiness estimation for safety driving using independent component analysis," in IEEE Transactions on Circuits and Systems.

[17] J. Vicente, P. Laguna, A. Bartra and R. Bailón, "Detection of driver's drowsiness by means of HRV analysis," 2011 Computing in Cardiology, 2011, pp. 89-92

[18] Tianyi Hong and Huabiao Qin, "Drivers drowsiness detection in 
embedded system," 2007 IEEE International Conference on Vehicular Electronics and Safety, 2007, pp. 1-5

[19] W. Zhang, B. Cheng and Y. Lin, "Driver drowsiness recognition based on computer vision technology," in Tsinghua Science and Technology, vol. 17, no. 3, pp. 354-362

[20] C. Li and J. Lin, "Random Body Movement Cancellation in Doppler Radar Vital Sign Detection," in IEEE Transactions on Microwave Theory and Techniques, vol. 56, no. 12, pp. 31433152

[21] Park, J., Kwon, H., Kang, S., \& Lee, Y. (2018, October). The effect of binaural beat-based audiovisual stimulation on brain waves and concentration. In 2018 International Conference on
Information and Communication Technology Convergence (ICTC) (pp. 420-423). IEEE.

[22] Solca, M., Mottaz, A., \& Guggisberg, A. G. (2016). Binaural beats increase interhemispheric alpha-band coherence between auditory cortices. Hearing research, 332, 233-237.

(C) 2021 by the In Chung Kyo and Min Byung Chan. Submitted for possible open access publication under the terms and conditions of the Creative Commons Attribution (CC BY) license

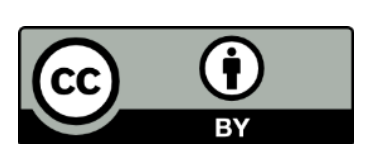

(http://creativecommons.org/license s/by/4.0/). 